\title{
SOME CARDINALITY PROPERTIES OF A HYPERSPACE WITH THE LOCALLY FINITE TOPOLOGY
}

\author{
A. BELLA
}

(Communicated by Doug W. Curtis)

\begin{abstract}
Estimates of various cardinal functions defined on a hyperspace equipped with the locally finite topology are given.
\end{abstract}

The locally finite topology on a hyperspace is the natural generalization of the well-known Vietoris or finite topology. Recently some authors (see [1 and 9]) have investigated its properties finding interesting relationships with the Hausdorff metric topology, when the base space is metrizable, and the uniform topology, when the base space is normal. In this paper we are concerned with some cardinality properties of a hyperspace with the locally finite topology. Specifically we provide estimates of various cardinal functions defined on it. Some results of the same kind relative to the finite case can be found in $[\mathbf{3}, \mathbf{6}$, and $\mathbf{8}]$.

For notation and terminology we refer to [5]. Each cardinal number is assumed to be an initial ordinal. As usual, given two cardinal numbers $\lambda, \not{k}, \not<>$ is defined as $\sup \left\{\ell^{\mu}: \mu<\lambda\right\} .|S|$ denotes the cardinality of the set $S$ and $\mathscr{P}(S)$ its powerset. $[S]^{k}\left([S]^{<k}\right.$ or $\left.[S]^{\leq k}\right)$ is the collection of all subsets of $S$ having cardinality $\not k$ (less than $k$, or less or equal to $k$ ).

Throughout the paper $X$ always denotes a topological $T_{1}$ space. $c(X), d(X)$, $\pi(X), w(X), L(X), \chi(X)$ and $\pi_{\chi}(X)$ denote, respectively, the cellularity, and density, the $\pi$-weight, the weight, the Lindelöf number, the character and the $\pi$ character of $X . \chi(A, X)$ denotes the character of the subset $A$ of $X$.

For definitions and more details concerning these cardinal functions we refer to the Juhász's book [7]. All other cardinal functions used here will be explicitly defined.

DEFINITION 1. The weak covering number of $X$, denoted by $\operatorname{wc}(X)$, is the smallest infinite cardinal number $k$ such that every open cover of $X$ has a subfamily of cardinality at most $\not k$ whose union is dense in $X$.

DEFINITION 2. The pseudocompactness number of $X$, denoted by $p(X)$, is the smallest infinite cardinal number $k$ such that every locally finite family of open subsets of $X$ has cardinality at most $k$.

To formulate some of our results in a little stronger way than using exponentiation by $p(X)$ or $L(X)$, we need also the following (see $[7,1.22]$ ):

DEFINITION $2^{\prime} . \hat{p}(X)$ is the smallest infinite cardinal number $k$ such that in $X$ there exists no locally finite family of open sets of cardinality $\boldsymbol{k}$.

Clearly $X$ is pseudocompact if and only if it is Tychonov and $\hat{p}(X)=\aleph_{0}[\mathbf{5}, \mathrm{p}$. 263].

Received by the editors June 9, 1985 and, in revised form, September 10, 1985.

1980 Mathematics Subject Classification (1985 Revision). Primary 54A25, 54B20. 
DEFINITION 3. $\hat{L}(X)$ is the smallest infinite cardinal $k$ such that for any open cover $X$ there exists a subcover cardinality less than $\mathscr{k}$. $\exp (X)$ denotes the collection of all nonempty closed subsets of $X$, i.e. the hyperspace of $X$.

Given a family $\mathscr{S} \subset \mathscr{P}(X)$, we indicate by $\langle\mathscr{S}\rangle$ the subset of $\exp (X)$ consisting of all $A \in \exp (X)$ for which $A \subset \bigcup \mathscr{S}$ and $A \cap S \neq \varnothing$ for any $S \in \mathscr{S}$. The locally finite (finite) topology on $\exp (X)$ is constructed taking as a base the sets of the form $\langle\mathscr{U}\rangle$, where $\mathscr{U}$ is a locally finite (finite) family of open subsets of $X$.

From now on $\exp (X)$ will be considered endowed with the locally finite topology. Before stating our first theorem we recall a well-known result of Erdös and Rado on partition calculus.

PROPOSITION (SEE E.G. [10]). If $\not k$ is an infinite cardinal, then $\left(2^{k}\right)^{+} \rightarrow$ $\left[\mathfrak{h}^{+}\right]_{k}^{2}$.

This standard combinatorial notation means that, whenever $S$ is a set with $|S|>2^{k}$ and $[S]^{2}=\bigcup_{\alpha \in k} P_{\alpha}$, there exist some $\alpha_{0} \in k$ and some $S_{0} \in \mathscr{P}(S)$ such that $\left[S_{0}\right]^{2} \subset P_{\alpha_{0}}$ and $\left|S_{0}\right|>\mathscr{k}$.

LEMMA 1. $p(X) \leq \mathrm{wc}(X) \leq \min \{c(X), L(X)\}$.

ProOF. See [4, Proposition 1.2].

THEOREM 1. $c(\exp (X)) \leq 2^{c(X)}$.

ProOF. Let $k=c(X)$. Assume by contradiction that $c(\exp (X))>2^{k}$. Let $E$ be the collection of all nonempty, closed, discrete subsets of $X$ having cardinality not exceeding $\not k$. Since by Lemma $1, p(X) \leq \not h$ it follows that $E$ is dense in $\exp (X)$ and consequently $c(E)>2^{k}$. Now define a function $\varphi: X^{k} \rightarrow \mathscr{P}(X)$ by sending each point $x$ of $X^{k}$, of course understood as a function from $\ell$ into $X$, in $\{x(\alpha): \alpha \in \not k\}$.

Denote by $\tau(X)$ the topology of $X$ and let $\mathscr{F}$ be the collection of all functions from $h$ into $\tau(X) \backslash\{\varnothing\}$. It is easy to see that the family $\left\{\prod_{\alpha \in k} f(\alpha): f \in \mathscr{F}\right.$ and $\{f(\alpha): \alpha \in k\}$ locally finite $\}$ can be regarded as a base for a topology on $X^{k}$. We indicate the space so obtained by $X_{l f}^{k}$. Equipping $\varphi^{-1}(E)$ with the subspace topology induced from $X_{l f}^{k}$, the restriction $\varphi_{\mid \varphi^{-1}(E)}$ becomes a continuous function from $\varphi^{-1}(E)$ onto $E$ and hence $c\left(\varphi^{-1}(E)\right)>2^{k}$. To verify the continuity of the function $\varphi_{\mid \varphi^{-1}(E)}$ fix a point $x \in \varphi^{-1}(E)$ and let $R$ be a neighborhood of $\varphi_{\mid \varphi^{-1}(E)}(x)$ in $E$. Since $E$ has the induced topology from $\exp (X)$, there exists a locally finite family $U$ of open subsets of $X$ such that $\varphi_{\mid \varphi^{-1}(E)}(x) \in\langle\mathscr{U}\rangle \cap E \subseteq R$. For any $\alpha \in \mathscr{k}$ let $\mathscr{U}_{\alpha}=\{U: U \in \mathscr{U}, x(\alpha) \in U\}$. $\mathscr{U}_{\alpha}$ is finite and hence the set $V_{\alpha}=\bigcap \mathscr{U}_{\alpha}$ is an open set containing $x(\alpha)$. We claim that the family $\left\{V_{\alpha}, \alpha \in \not{k}\right\}$ is locally finite. Indeed let $p \in X$ and let $W$ be a neighborhood of $p$ meeting only finitely many members of $\mathscr{U}$. Let $\Lambda=\left\{\alpha: \alpha \in \mathscr{k}, W \cap V_{\alpha} \neq \varnothing\right\}$. For any $\alpha \in \Lambda$, $W$ meets each members of $\mathscr{U}_{\alpha}$ and therefore the set $\bigcup_{\alpha \in \Lambda} \mathscr{U}_{\alpha}$ must be finite. Since for any $\alpha \in \Lambda V_{\alpha}$ is the intersection of a subfamily of $\bigcup_{\alpha \in \Lambda} \mathscr{U}_{\alpha}$ it follows that also the set $\left\{V_{\alpha}, \alpha \in \Lambda\right\}$ is finite and thus the family $\left\{V_{\alpha}: \alpha \in \ell\right\}$ is locally finite. The set $\prod_{\alpha \in \ell} V_{\alpha}$ is open $X_{l f}^{k}$ and consequently the set $\prod_{\alpha \in \ell} V_{\alpha} \cap \varphi^{-1}(E)$ is an open neighborhood of $x$ in $\varphi^{-1}(E)$. It is easy to see that $\left\langle V_{\alpha}: \alpha \in \mathscr{k}\right\rangle \subset\langle\mathscr{U}\rangle$ and finally we have $\left.\varphi_{\mid \varphi^{-1}(E)} \subset \prod_{\alpha \in \ell} V_{\alpha} \cap \varphi^{-1}(E)\right) \subset\left\langle V_{\alpha}: \alpha \in \not l\right\rangle \cap E \subset\langle\mathscr{U}\rangle \cap E \subset R$, and this completes the proof of the continuity of $\varphi_{\mid \varphi^{-1}(E)}$. Furthermore $\varphi^{-1}(E)$ is 
dense in $X_{l f}^{k}$ and consequently $c\left(X_{l f}^{k}\right)>2^{k}$. Let $\left\{U_{s}\right\}_{s \in S}$ be a family of pairwise disjoint nonempty open subsets of $X_{l f}^{k}$ such that $|S|>2^{k}$. Without any loss of generality we can suppose that for, any $s \in S, U_{s}$ is a member of the previously described base defined by some $f^{s} \in \mathscr{F}$.

For any $\alpha \in \mathcal{R}$ let $P_{\alpha}=\left\{\{s, t\}: s, t \in S\right.$ and $\left.f^{s}(\alpha) \cap f^{t}(\alpha)=\varnothing\right\}$. It is clear that $[S]^{2}=\bigcup_{\alpha \in k} P_{\alpha}$ and so by the Erdös-Rado's theorem there exist some $\alpha_{0} \in \mathscr{k}$ and some $S_{0} \in \mathscr{P}(S)$ such that $\left[S_{0}\right]^{2} \subset P_{\alpha_{0}}$ and $\left|S_{0}\right|>k$. The family $\left\{f^{s}\left(\alpha_{0}\right): s \in S_{0}\right\}$ consists of more than $\not k$ pairwise disjoint nonempty open subsets of $X$. This is a contradiction and the proof is complete.

Note that taking as $X$ a discrete space we obtain $c(\exp (X))=2^{c(X)}=2^{|X|}$, while taking as $X$ the Alexandröff compactification of a discrete space we obtain $c(\exp (X))=c(X)=|X|$.

REMARK. The topology of $X_{l f}^{k}$ clearly lies between the Tychonov and the box topology.

It differs from the Tychonov topology whenever $k$ is infinite and $|X|>1$. It differs from the box topology at least when $X$ is not a discrete space and $k \geq$ $\pi \chi(p, X)$ for some nonisolated point $p \in X$. To check the last assertion let $\left\{U_{\alpha}\right\}_{\alpha \in \lambda}$, $\lambda \leq k$, be a local $\pi$-base at $p$ and define a function $f: k \rightarrow \tau(X)$ in such a way that $f(\alpha)=U_{\alpha}-\{p\}$ for any $\alpha \in \lambda$, and $f(\alpha)=X-\{p\}$ for any $\alpha \in \not k-\lambda$. Put $U_{f}=\prod_{\alpha \in k} f(\alpha)$ and let $\varphi$ and $E$ defined as in the above theorem. $U_{f}$ is open in the box topology, but $U_{f} \cap \varphi^{-1}(E)=\varnothing$ because for any $x \in U_{f}$ we have $p \in \overline{\varphi(x)} \backslash \varphi(x)$. Since $\varphi^{-1}(E)$ is dense in $X_{l f}^{k}$ the two topologies are different.

THEOREM 2. $w(\exp (X)) \leq w(X)^{<\hat{L}(X)}$.

ProOF. Let $\mathscr{B}$ be a base of $X$ such that $|\mathscr{B}| \leq w(X)$. We claim that the set $\left\{\left\langle\left\{\bigcup \mathscr{G}_{1}\right\} \bigcup \mathscr{G}_{2}\right\rangle: \mathscr{G}_{1}, \mathscr{G}_{2} \in[\mathscr{B}]^{<\hat{L}(X)}\right\}$ contains a base for $\exp (X)$ and this fact concludes the proof because such a set has cardinality not exceeding $w(X)^{<\hat{L}(X)}$. Indeed let $\mathscr{U}$ be a locally finite family of open subsets of $X$ and $A \in\langle\mathscr{U}\rangle$ for some $A \in \exp (X)$. Since $A$ is closed, there exists some $\mathscr{G}_{1} \in[\mathscr{B}]^{<\hat{L}(X)}$ such that $A \subset \bigcup \mathscr{G}_{1} \subset \bigcup \mathscr{U}$. For any $U \in \mathscr{U}$ choose $B_{U} \in \mathscr{B}$ such that $B_{U} \subset U$ and $A \cap B_{U} \neq \varnothing$ and let $\mathscr{G}_{2}$ be the set so obtained. We have $|\mathscr{U}|<\hat{p}(X) \leq \hat{L}(X)$ and hence $\mathscr{G}_{2} \in[\mathscr{B}]^{<\hat{L}(X)}$. Since $A \in\left\langle\left\{\cup \mathscr{G}_{1}\right\} \cup \mathscr{G}_{2}\right\rangle \subset\langle\mathscr{U}\rangle$ and $\left\{\cup \mathscr{G}_{1}\right\} \cup \mathscr{G}_{2}$ is locally finite we are done.

Assuming the normality of the space under consideration, the inequality in the previous theorem can be sharpened.

THEOREM 3. If $X$ is normal, then $w(\exp (X)) \leq|Z(X)|^{<\hat{p}(X)}$, where $Z(X)$ is the family of all zero subsets of $X$.

ProOF. Let $\mathscr{B}$ be the family of all cozerosubsets of $X$. We claim that the family $\langle\mathscr{G}\rangle: \mathscr{G} \in[\mathscr{B}]^{<\hat{p}(X)}$ contains a base for the topology of $\exp (X)$. Indeed, let $\mathscr{U}$ be a locally finite family of open subsets of $X$ and $A \in\langle\mathscr{U}\rangle$ for some $A \in \exp (X)$. For any $U \in \mathscr{U}$ choose an element $B_{U} \in \mathscr{B}$ such that $B_{U} \subset U$ and $B_{U} \cap A \neq \varnothing$. By the normality of $X$ there exists an element $B \in \mathscr{B}$ such that $A \subset B \subset \cup \mathscr{U}$. Letting $\mathscr{G}=\{B\} \cup\left\{B_{U}: U \in \mathscr{U}\right\}$, it is clear that $\mathscr{G}=[\mathscr{B}]^{\langle\hat{p}(X)}$ and $A \in\langle\mathscr{G}\rangle \subset\langle\mathscr{U}\rangle$. Since $|\mathscr{B}|=|Z(X)|$ we obtain $w(\exp (X)) \leq|Z(X)|^{<\hat{p}(X)}$. 
COROLlaRY 1. If $X$ is normal, then $w(\exp (X)) \leq w(X)^{\mathrm{wc}(X)}$.

ProOF. Let $C(X)$ be the set of all real valued continuous functions on $X$. In [4] it is proved that $|C(X)| \leq w(X)^{\mathrm{wc}(X)}$ and, moreover, $|Z(X)| \leq|C(X)|$. By Lemma 1 , and Theorem 3 we obtain $w(\exp (X)) \leq|Z(X)|^{<\hat{p}(X)} \leq\left(w(X)^{\mathrm{wc}(X)}\right)^{<\hat{p}(X)} \leq$ $w(X)^{\mathrm{wc}(X) \cdot p(X)}=w(X)^{\mathrm{wc}(X)}$.

Let $S$ be a subset of $X$. Recall that an exterior $\pi$-base for $S$ is a family $\mathscr{B}$ of open subsets of $X$ such that, for any $B \in \mathscr{B}, B \cap S \neq \varnothing$ and, for any open subset $U$ of $X$ such that $U \cap S \neq \varnothing$, there exists some $B \in \mathscr{B}$ for which $B \subset U$.

The exterior $\pi$-weight of $S$, denoted by $\pi(S, X)$, is defined as the smallest infinite cardinal $k$ such that there exists an exterior $\pi$-base for $S$ having cardinality not exceeding $\not h$. Clearly, $\pi(X, X)=\pi(X)$.

THEOREM 4. $\chi(A, \exp (X)) \leq \chi(A, X) \cdot \pi(A, X)^{<\hat{p}(A)}$.

ProOF. Let $\mathscr{B}$ be a fundamental system of neighborhoods of $A$ in $X$ such that $|\mathscr{B}| \leq \chi(A, X)$ and $\mathscr{D}$ an exterior $\pi$-base for $A$ such that $|\mathscr{D}| \leq \pi(A, X)$. As in the previous theorem we can verify that the family $\{\langle\{B\} \cup \mathscr{G}\rangle: B \in \mathscr{B}$ and $\left.\mathscr{G} \in[\mathscr{D}]^{<\hat{p}(A)}\right\}$ contains a fundamental system of neighborhoods of the point $A$ in $\exp (X)$. We only have to note that, for any locally finite family $\mathscr{U}$ of open subsets of $X$ such that $A \in\langle\mathscr{U}\rangle$, the inequality $|\mathscr{U}|<\hat{p}(A)$ holds. Indeed, the family $\mathscr{V}=\{U \cap A: U \in \mathscr{U}\}$ satisfies $|\mathscr{V}|<\hat{p}(A)$ and, by the locally finiteness of $\mathscr{U}$ the set $\mathscr{U}_{V}=\{U: U \in \mathscr{U}$ and $U \cap A=V\}$ is finite for any $V \in \mathscr{V}$. Since $\mathscr{U}=\bigcup_{V \in \mathscr{V}} \mathscr{U}_{V}$ we clearly obtain $|\mathscr{U}|<\hat{p}(A)$.

A similar result is the following

THEOREM 5. $\pi \chi(A, \exp (X)) \leq \pi(A, X)^{<\hat{p}(A)}$.

It can be easily seen that $\chi(A, X) \leq[|A| \cdot \chi(X)]^{|A|}=\chi(X)^{|A|}$ and $\pi(A, X) \leq$ $[d(A) \chi(X)]^{d(A)}=\chi(X)^{d(A)}$. Thus we obtain the next two corollaries, using also the fact that $p(A) \leq d(A)$.

COROllary 2. $\chi(A, \exp (X)) \leq \chi(X)^{|A|}$.

Corollary $3 . \pi \chi(A, \exp (X)) \leq \chi(X)^{d(A)}$.

The proof of the next two theorems is rather straightforward and will be left to the reader.

THEOREM 6. $d(\exp (X)) \leq d(X)^{<\hat{p}(X)}$.

THEOREM $7 . \pi(\exp (X)) \leq \pi(X)^{<\hat{p}(X)}$.

To conclude, we consider another cardinal function.

DEFINITION 4. Let $X$ be a space with no isolated points. The Novak number of $X$ denoted by $n(X)$ is the smallest cardinal number $k$ such that $X$ can be covered by $k$ nowhere dense sets.

For further information concerning this cardinal function see [2].

Before looking at the Novak number of $\exp (X)$, we have to verify its definability.

LEMMA 2. If $X$ has no isolated point, then $\exp (X)$ also has no isolated point.

PROOF. It suffices to show that every nonempty open subset of $\exp (X)$ of the form $\langle\mathscr{U}\rangle$ contains at least two points. Choose a point $p$ belonging to some $U \in \mathscr{U}$ 
and let $\mathscr{U}_{p}=\{U: U \in \mathscr{U}$ and $p \in U\}$. As $\mathscr{U}$ is locally finite $\bigcap \mathscr{U}_{p}$ is an open neighborhood of $p$. Since $p$ is not isolated there exists some $q \in \bigcap \mathscr{U}_{p}-\{p\}$. For any $U \in \mathscr{U} \backslash \mathscr{U}_{p}$ choose a point in it and let $A$ be the set so obtained. It is clear that $A \cup\{p\}$ and $A \cup\{q\}$ are two distinct points of $\exp (X)$ belonging to $\langle\mathscr{U}\rangle$.

THEOREM 8. If $X$ has no isolated point, then $n(\exp (X)) \leq n(X)$.

ProOF. Let $\mathscr{k}=n(X)$ and let $\left\{U_{\alpha}\right\}_{\alpha \in k}$ be a family of dense open subsets of $X$ such that $\bigcap_{\alpha \in k} U_{\alpha}=\varnothing$. It is easily seen that also the family $\left\{\left\langle U_{\alpha}\right\rangle\right\}_{\alpha \in k}$ is a family of dense open subsets of $\exp (X)$ with an empty intersection. This clearly means that $n(\exp (X)) \leq k$.

We do not know whether there exists some space $X$ for which $n(\exp (X))<n(X)$.

ACKNOWLEDGEMENTS. The author wishes to thank the referee for his comments.

\section{REFERENCES}

1. G. Beer, C. J. Himmelberg, K. Prikry and F. Van Vleck, The locally finite topology on $2^{X}$, Proc. Amer. Math. Soc. 101 (1987), 163-172.

2. A. Bella, Some remarks on the Novak number, Proc. 6th Prague Topological Sympos. (Prague, August 1986) Heldermann-Verlag, Berlin, 1988, 43-48.

3. M. M. Coban, On exponential topology, Soviet Math. Dokl. 10 (1969), 597-600.

4. W. W. Comfort and A. W. Hager, Estimates for the number of real-valued continuous functions, Trans. Amer. Math. Soc. 150 (1970), 619-631.

5. R. Engelking, General topology, Polish Scientific Publishers, Warsaw, 1977.

6. J. Ginsburg, Contributions to the theory of hyperspaces, Doctoral dissertation, University of Manitoba, 1975.

7. I. Juhasz, Cardinal functions in topology - Ten years later, Math Centre Tract 123, Amsterdam, 1980.

8. V. I. Malyhin, On tightness and Suslin number in $\exp (X)$ and in a product of spaces, Soviet Math. Dokl. 13 (1972), 2.

9. S. Naimpally and P. Sharma, Fine uniformity and the locally finite hyperspace topology, Proc. Amer. Math. Soc. (to appear).

10. M. E. Rudin, Lectures on set-theoretical topology, CBMS Regional Conf. Ser. in Math., No. 23, Amer. Math. Soc., Providence, R. I., 1975.

Dipartimento di Matematica, Università di Catania, viale A. Doria 6, 95125 CATANia, Italy 